\title{
Analysis of Vibration and Noise for the Powertrain System of Electric Vehicles under Speed-Varying Operating Conditions
}

\author{
Chenghao Deng $\mathbb{D},{ }^{1,2}$ Qingpeng Deng $\mathbb{D},{ }^{2}$ Weiguo Liu, ${ }^{2}$ Cheng Yu, ${ }^{2}$ Jianjun Hu, ${ }^{1}$ \\ and Xiaofeng $\mathrm{Li}^{3}$ \\ ${ }^{1}$ School of Automotive Engineering, Chongqing University, Chongqing 400040, China \\ ${ }^{2}$ Chongqing Chang'an New Energy Automobile Technology Co. Ltd., Chongqing 401120, China \\ ${ }^{3}$ Chongqing Deyin Technology Co. Ltd., Chongqing 400050, China
}

Correspondence should be addressed to Chenghao Deng; dengch@changan.com.cn and Qingpeng Deng; dqp_2017@163.com

Received 10 October 2020; Revised 1 November 2020; Accepted 5 November 2020; Published 23 November 2020

Academic Editor: Yong Chen

Copyright (c) 2020 Chenghao Deng et al. This is an open access article distributed under the Creative Commons Attribution License, which permits unrestricted use, distribution, and reproduction in any medium, provided the original work is properly cited.

\begin{abstract}
Whine noise from the electric powertrain system of electric vehicles, including electromagnetic noise and gear-meshing noise, significantly affects vehicle comfort and has been getting growing concern. In order to identify and avoid whine problems as early as possible in the powertrain development process, this paper presents a vibration and noise simulation methodology for the electric powertrain system of vehicles under speed-varying operating conditions. The electromagnetic forces on the stator teeth of the motor and the bearing forces on the gearbox for several constant-speed operating conditions are obtained first by electromagnetic field simulation and multi-body dynamic simulation, respectively. Order forces for the speed-varying operating condition are generated by interpolation between the obtained forces, before they are applied on the mechanical model whose natural modes have been calibrated in advance by tested modes. The whine noise radiated from the powertrain is then obtained based on acoustic boundary element analysis. The simulated bearing forces indicate that the overlooking of the motor torque ripple does not result in significant loss in simulation accuracy of electromagnetic noise. The simulation results and tested data show good consistency, with the relative frequency deviation of local peaks being less than $8 \%$ and the error of the average sound pressure level (SPL) being mostly below $10 \mathrm{~dB}(\mathrm{~A})$.
\end{abstract}

\section{Introduction}

The electric vehicle industry has achieved rapid development in recent years. The high-frequency electromagnetic and gear whine noise emitted from the electric powertrain system could significantly affect driving comfort and has become an important noise, vibration, and harshness $(\mathrm{NVH})$ problem of electric vehicles. The motor and reducer are two main vibration and noise sources of the electric powertrain system. The electromagnetic forces of the motor and the gearmeshing forces of the reducer could cause structural vibration and whine noise that shows obvious order characteristics.

The integration design of the electric drive system has become a technological trend, which means that more functional units, such as the motor, reducer, motor control unit, and power supply, are integrated into just one drive unit, i.e., the electric powertrain. The integration of the electric drive system can significantly reduce the volume, weight, and cost of the powertrain system and hence gain competitive advantages in the market place. As a result, the physical boundaries between components turn increasingly vague, and the mechanical coupling between the component structures become stronger, which introduces challenges to $\mathrm{NVH}$ analysis and control [1]. The generation mechanism of powertrain whine noise is consistent with that of the motor and reducer [2]. However, the coupling effect between the components will significantly affect the characteristics of whine noise. For instance, the tangential electromagnetic forces acting on the stator teeth, which can be ignored in the noise analysis of a standalone motor, turn 
to be nonnegligible in the NVH simulation of the integrated powertrain, as the tangential electromagnetic forces could excite the reducer housing to vibrate and radiate noise [3]. Fang and Zhang [4] analyzed the vibration characteristics of the electric drive system through simulation and test, revealing that the motor, reducer, and controller, due to the coupling phenomenon, must be considered as an indivisible whole in the NVH analysis. Vibration and sound simulation based on computer-aided engineering (CAE) is an important approach for analyzing and optimizing electric powertrain noise, especially in the early phase of design. Harris et al. [5] introduced a CAE method to optimize the whine problem caused by gear-meshing excitation. The dynamic meshing force at the contact point of gears is reduced by changing the geometry of the rim and web of the gears. Yu et al. [6, 7] established a finite element (FE) model of the electric drive assembly system to predict its vibration. The simulation model reflects part of the frequency characteristics of vibration, but quantitative evaluation on the vibration simulation accuracy is not provided. The key challenge of $\mathrm{NVH}$ simulation for the highly integrated electric powertrain system is to efficiently calculate the whine noise caused by various types of excitations, such as electromagnetic forces and gear-meshing forces, within the whole speed range and with good simulation accuracy. However, the NVH simulation method for integrated electric powertrain with both satisfactory accuracy and efficiency is rarely reported.

In this paper, NVH simulation analysis for an integrated electric powertrain system under electromagnetic and gear-meshing excitations is performed. The electromagnetic forces on the stator teeth and gear-meshing forces acting on the bearings in the time domain for several constant-speed operating conditions are obtained first by electromagnetic simulation and multi-body dynamic simulation. Forces in the frequency domain are then obtained by performing fast Fourier transform (FFT). Cubic spline interpolation is utilized to obtain the order forces under the speed-varying condition, which significantly cuts down the time required for multicondition force simulation. In order to ensure the simulation accuracy, the material parameters of the motor stator are calibrated by performing modal correlation analysis of the tested modes and the simulated modes before the FE model is used for any dynamic simulation, including the multi-body analysis and vibration simulation. The acoustic transfer vector (ATV) from the surface vibration of the powertrain housing to the sound pressure of the observing point is calculated by the acoustic FE analysis. Finally, the radiated sound pressure is calculated by using the obtained housing vibration velocity and ATV. Based on the simulation model, the influence of the motor torque ripple on the whine noise is evaluated. The effectiveness of the simulation method is verified by using tested results. The influence of the stator breathing mode on the 48-order whine noise is revealed. The main contribution of the paper lies in the presentation of an $\mathrm{NVH}$ simulation method for the electric powertrain system with satisfactory accuracy and efficiency, which makes it possible to identify and avoid whine problems at early stages of powertrain development.

\section{Process of Vibration and Sound Analysis}

When the electric vehicle is speeding up or decelerating, harmonic excitation forces with order characteristics in the electric drive system excite the powertrain housing to vibrate and radiate noise into the air. There are two main types of harmonic excitation forces responsible for whine noise, i.e., electromagnetic excitation loads and gear-meshing forces. The electromagnetic loads mainly consist of two parts, including the electromagnetic forces on the stator teeth and the torque ripple on the rotor [8-10]. The former acts on the stator structure directly, while the latter acts on the rotor shaft which transmits the pulsating harmonic load to the powertrain housing through the bearings of the drive system [11]. Gear-meshing forces are the dynamic loads produced by the interaction between meshing gears, which can also be transmitted to the powertrain housing through the bearings. In this paper, the time-domain electromagnetic loads under a couple of constant-speed conditions are obtained by 2-dimensional (2D) electromagnetic field simulation in the software Maxwell. The time-domain forces on the bearings under the constant-speed conditions are obtained through multi-body dynamic simulation. Then, the order loads are generated by the interpolation algorithm after the forces of the constant-speed conditions have been obtained. The normal vibration velocity of the powertrain housing is obtained by using FE analysis by applying the order forces onto the structural model. The acoustic FE module in the commercial software Virtual Lab is used to calculate the acoustic transfer vector (ATV) from the surface vibration velocity of the powertrain housing to sound pressure at the acoustic observing positions. At last, the sound pressure levels (SPLs) of the observing positions can be calculated by the surface vibration velocity of the powertrain housing and the ATV.

NVH simulation error of the electric powertrain system is affected by a number of factors, among which the modeling accuracy of the structural modes is a crucial one. On the one hand, the modeling accuracy of the structural modes determines the accuracy of the multi-body dynamic simulation, which means it will affect the computed results of the bearing forces. On the other hand, the simulated results of forced vibration are significantly affected by the accuracy of structural modes. The key challenge in modeling the powertrain structure lies in the treatment of the stator. The stator core is composed of compacted silicon steel sheets, which shows material anisotropy and parameter uncertainty. These characteristics make it quite difficult to model the core accurately. In addition, the stator windings have similar characteristics. In order to model the stator as accurate as possible, modal correlation analysis for tested modes and simulated modes [12-14] is performed to calibrate the stator parameters before the FE model is used for multi-body dynamic simulation and vibration analysis. The complete process of the NVH simulation analysis is illustrated in Figure 1. 


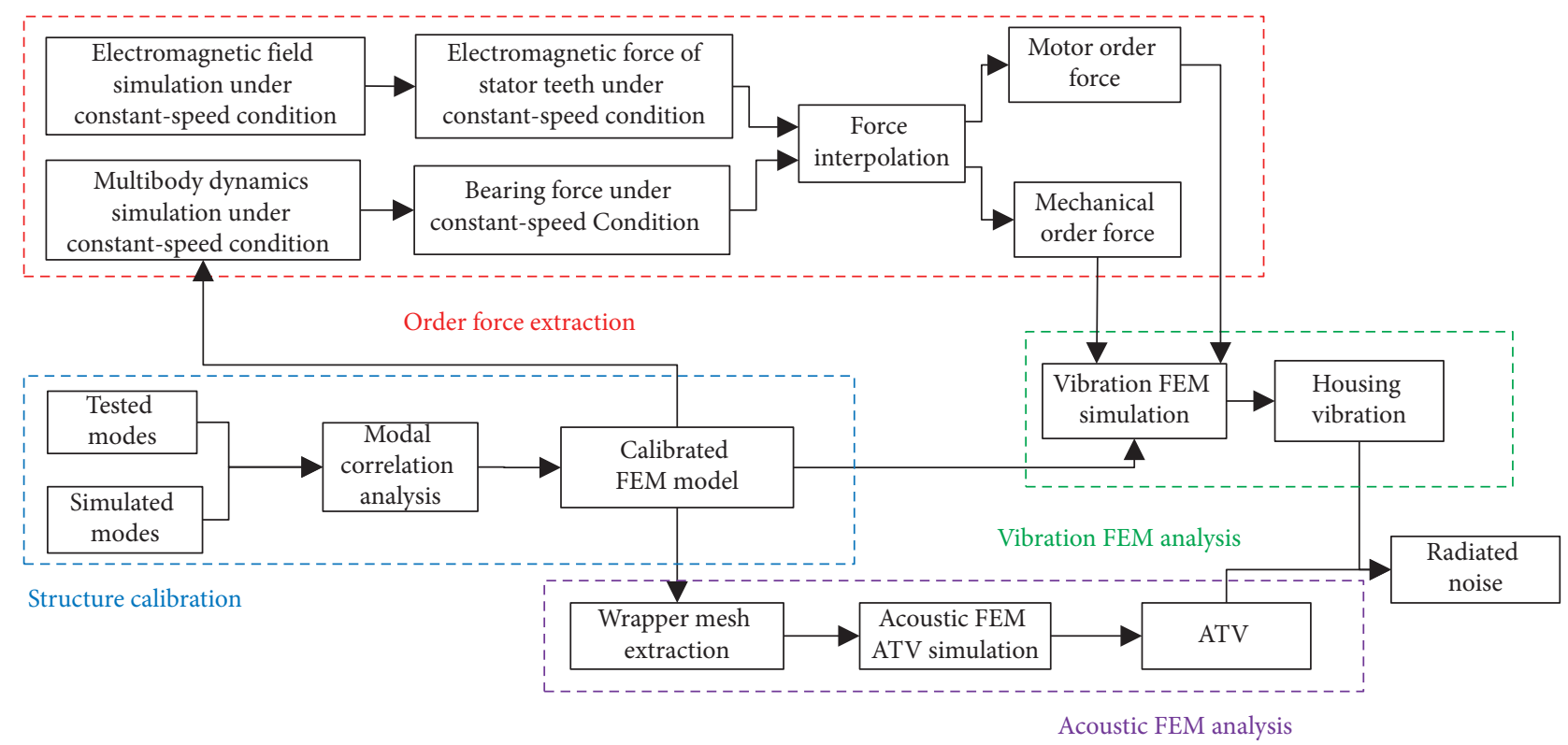

FIGURE 1: Process of vibration and sound analysis.

\section{FE Modeling and Calibration for the Powertrain System}

In Figure 2, the 3D structural model of powertrain $\mathrm{NVH}$ simulation is illustrated. The powertrain system consists of a motor, a motor controller, and a reducer, and it connects to the vehicle body through the suspensions.

The difficulty of FE modeling lies in the construction of the stator model. Under the constant-speed condition, the air-gap electromagnetic force is a periodic function of time and circumferential angle. As a result, under the speedvarying condition, the air-gap force shows order characteristics in both the frequency domain and the wavenumber domain. The air-gap electromagnetic load can be considered as a superposition of a series of "force patterns" which can be regarded as a set of basis of the electromagnetic load and can be obtained by 2D Fourier transform (FT) to the air-gap electromagnetic load. Each force pattern has a specific spatial distribution and rotates circumferentially under a specific frequency. The magnitude of the electromagnetic noise is heavily dependent on the level of agreement between the "force patterns" and the stator modes. While the rotating frequency of a "force pattern" is close to a modal frequency and the shape of the "force pattern" matches well with the modal shape, the stator will experience strong resonance, radiating intense electromagnetic noise. In order to confirm the accuracy of simulation, an accurate FE model of stator structure is of great importance. Natural modes for the stator structure are tested in advance to conduct parameter calibration.

The calibration of the model is a process of optimizing model parameters, thus making the simulation model represent the actual dynamic characteristics of the structure and leading to good agreement between the simulation results and the test data regarding the modal shapes and frequencies. In general, there are two approaches in terms of

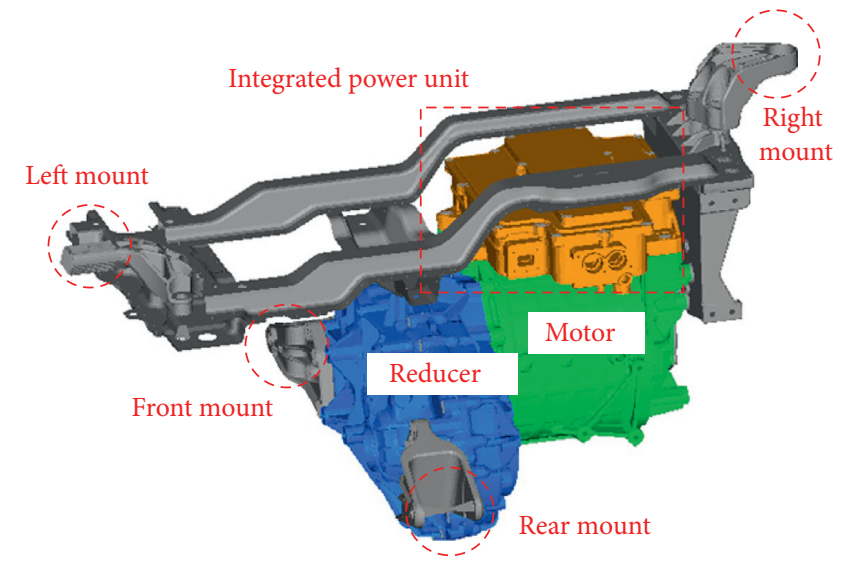

FIgURE 2: Structural model of the electric powertrain system.

calibration, namely, manual adjustment and modal correlation analysis. Manual adjustment needs to tune each physical parameter in order to achieve good agreement. Hence, this method heavily relies on personal experience and is difficult to implement for a complicated model. Modal correlation analysis can be carried out by using commercial software, for example, the correlation module in Virtual Lab, in which parameters can be optimized automatically after importing the tested modes into the software. In this paper, the latter method has been adopted.

The FE model of the stator is shown in Figure 3. The modeling method of the winding is revealed in the zoomin image of Figure 3. The winding is considered to be made up of two parts: the equivalent isolation layer and the equivalent winding. The former part is isotropic material with small elasticity modulus, and the latter is anisotropic material. The initial material parameters of the stator core and the equivalent winding are set according to [12]. The material for the isolation layer is polyimide with the 


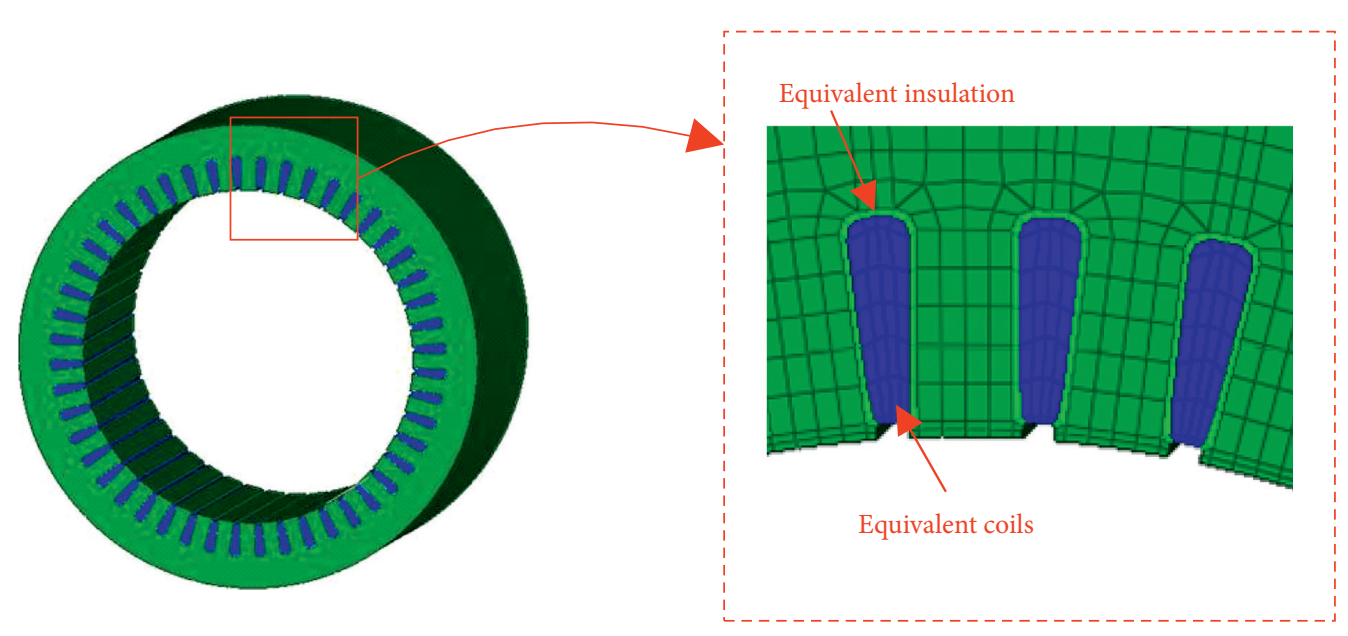

FIGURE 3: FE model of the stator core with winding.

default setting to be shown as follows: density $\rho=1.2 \mathrm{~g} / \mathrm{ml}$, elasticity modulus $E=3 \mathrm{GPa}$, and Poisson ratio $\mu=0.35$.

In the modal test, the frequency response functions are obtained under transient excitations. The stator is hung with an elastic slope, and a hammer is used to excite structural vibration. In total, 36 vibration-measuring points are distributed in the matrix along the stator surface, 3 circles with 12 test points equally spaced within one circle. Three axial acceleration sensors are used to acquire vibration signals. After the modal test, the results are fed to the correlation module of Virtual Lab to carry out correlation analysis and parameter optimization. In Figure 4, the comparison between simulated and tested modes is provided, showing the modal results with axial order $m=0$ and circumferential order $n$ being $0,2,3$, and 4, respectively. The discrepancy stands for the relative error between the computational modal frequency and experimental modal frequency. $\mathrm{MAC}=1$ means that the two modes are identical, while $\mathrm{MAC}=0$ denotes that the mode shapes are orthogonal. As can be seen in Figure 4, after parameter calibration, the FE model can represent the natural vibration characteristics accurately. The relative errors for the 4 modes are less than $6.4 \%$, with the correlation coefficient higher than 0.6. Apart from the stator, the material for other structural parts can be considered as isotropic, which will not be discussed in detail here.

\section{Simulation of Excitation Forces}

The NVH performance of the powertrain system depends on the running conditions. In most conditions, the vehicle velocity and motor torque are often transient under realworld driving. In general, the whole throttle acceleration and coasting deceleration are the two worst conditions in terms of powertrain whine noise for electric vehicles. Therefore, these two conditions are normally selected as the key conditions to be evaluated for powertrain NVH performance. Taking the whole throttle condition as an example, simulation of the order of vibration and noise has been carried out for the powertrain system. In order to obtain the order forces, the electromagnetic forces and bearing forces under constant-speed conditions are obtained at first, and then the order forces under the speed-varying condition can be calculated with interpolation.

4.1. Electromagnetic Forces under Constant-Speed Conditions. The powertrain system is equipped with a permanent magnet synchronous motor with 8 poles and 48 slots. FE analysis for the electromagnetic field in the software Maxwell has been performed to constant-speed conditions to obtain the electromagnetic forces. The simulation of the electromagnetic field is conducted from $1000 \mathrm{rpm}$ to the maximum speed, with a step of $1000 \mathrm{rpm}$. According to the previous research work [15], satisfactory simulation accuracy can be achieved when applying interpolation to electromagnetic force calculation with this step.

Instead of using the electromagnetic forces at the nodes of each tooth for subsequent interpolation and simulation directly, the equivalent concentrated electromagnetic forces for each tooth are adopted. The node forces on the tooth are equalized to a concentrated axial force and a concentrated tangential force, with the distribution effect of the electromagnetic loading in circumferential direction neglected. This approach brings in little deterioration in simulation accuracy but significantly reduces the amount of data to be processed, hence improving simulation efficiency. The 48 slots of the motor are evenly distributed, and the electromagnetic force is sampled in circumference at 48 points. According to the sampling theorem, when carrying out FFT, only the forces for the first 24 spatial orders can be recognized. When the concentrated forces are adopted for $\mathrm{NVH}$ calculation, the contribution of forces with spatial orders higher than $24(n>24)$ is ignored. Generally, the electromagnetic vibration of the motor is heavily dependent on the circumferential structural modes with low orders. As the order increases, the vibration amplitude of the mode decreases with a speed of $n^{4}$ [16]. In addition, as the force order increases by a multiple of the number of poles, the amplitude of the electromagnetic force shows a decreasing trend. Hence, the application of concentrated electromagnetic force has little influence on simulation accuracy. 


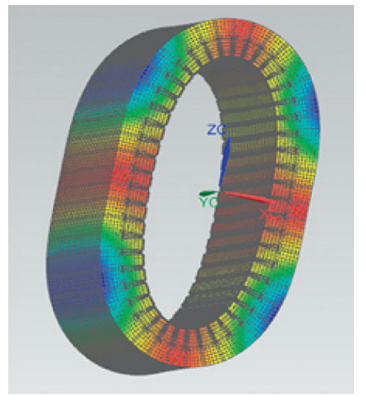

Simulation: $689.7 \mathrm{~Hz}$ Error: $4.2 \%$

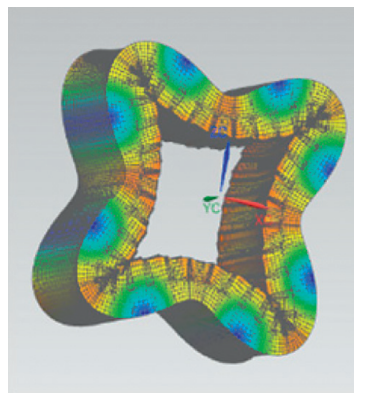

Simulation: $3329.5 \mathrm{~Hz}$ Error: $-6.4 \%$

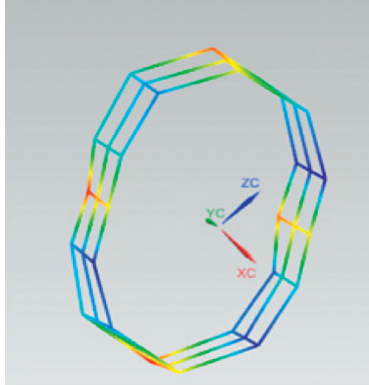

Test: $662 \mathrm{~Hz}$ MAC: 0.81

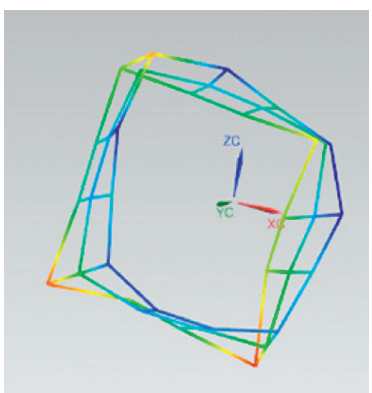

Test: $3557 \mathrm{~Hz}$

MAC: 0.60

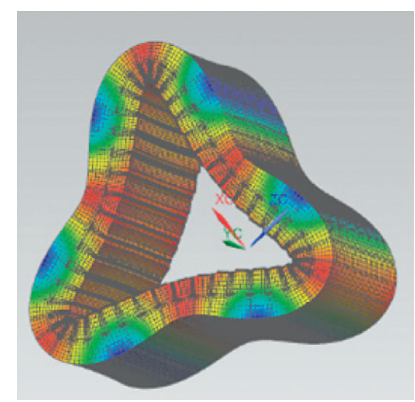

Simulation: $1837.1 \mathrm{~Hz}$

Error: $2 \%$

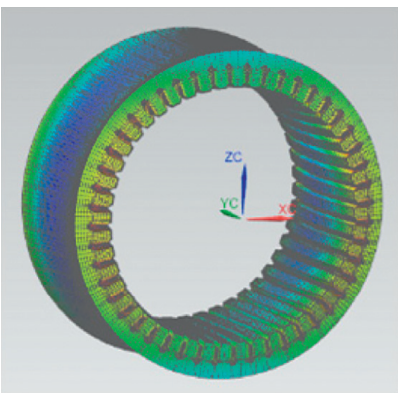

Simulation: $5491.8 \mathrm{~Hz}$

Error: $-3.1 \%$

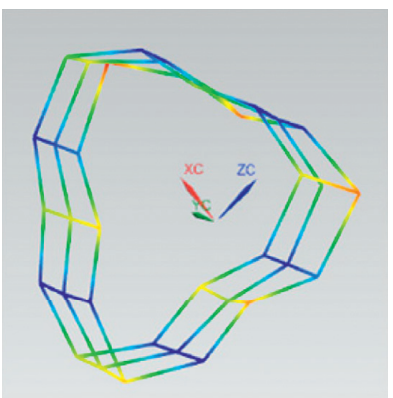

Test: $1801 \mathrm{~Hz}$

MAC: 0.87

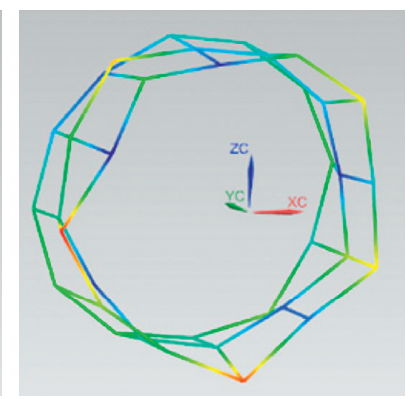

Test: $5667 \mathrm{~Hz}$ MAC: 0.83

FIgURE 4: Modal results of the stator core with winding.

4.2. Bearing Forces under Constant-Speed Conditions. The reducer is a one-ratio two-stage gear transmission system, and there are two pairs of helical gears in the gearbox for speed slowdown and torque increasing. In addition, there is a pair of differential gears. Dynamic forces generate during gear-meshing process, which is transmitted to the axles first and then to the powertrain housing via bearings. In this paper, multi-body dynamic simulation is performed to obtain the exciting forces at the bearings under constantspeed conditions.

According to research work $[17,18]$, the flexibility of the housing has influence on dynamic meshing force characteristics. Hence, the powertrain housing is considered as a flexible body in the multi-body dynamic model. Before feeding the FE model which has been calibrated in Section 3 to the multi-body dynamic model, modal condensation is used to reduce the degrees of freedom of the housing model, hence improving efficiency. Provided with the highest frequency of the noise of interest $f_{\text {max }}$, only the housing modes with natural frequency below $2 f_{\max }$ are retained. In Section 4.3 , the speed conditions selected for multibody dynamic simulation are introduced.

4.3. Order Forces under the Varying Speed Condition. In this section, the order forces under the acceleration condition can be obtained by applying interpolation. The notion "order" refers to how many times the frequency is referenced to the rotating frequency of the rotor. FFT is performed to obtain the frequency spectrums of the forces. In the end, force interpolation in the frequency domain between adjacent constant-speed conditions is performed. Cubic spline interpolation is used here, with the boundary condition at each endpoint being a not-a-knot boundary (the 3rd derivative at the endpoint equals that of the adjacent point).

The constant-speed conditions used for interpolation include speeds of two parts, with one part being the same as that used in electromagnetic force simulation and the other being additional speed conditions. The latter is included to take into account the influence of the natural vibration characteristics of the powertrain housing on the order bearing forces. Under each additional speed condition, the order frequency of the gear engagement matches well with one of the natural frequencies of the powertrain housing. Figure 5 illustrates how to select additional speed conditions. $f_{i}$ represents the modal frequency of the powertrain housing. The order lines intersect with the resonance frequency lines, and the speeds at the intersection points are selected as the additional speed conditions. For example, speeds $N_{i}^{1}$ and $N_{i}^{2}$ are included in force interpolation to take into account the coupling effects of the housing mode $f_{i}$. If the number of the housing modes within the frequency range of interest is $I$ and the number of force orders of interest is $q$, the number of additional speed conditions should be $I^{*} q$.

\section{Vibration and Noise Simulation}

5.1. Vibration Modeling and Loading. Before vibration simulation, the natural modes of the powertrain system should be computed by FE analysis. In this paper, the commercial software Nastran is used for the modal calculation, and then the modal results are imported into the software LMS Virtual Lab for force loading and vibration 


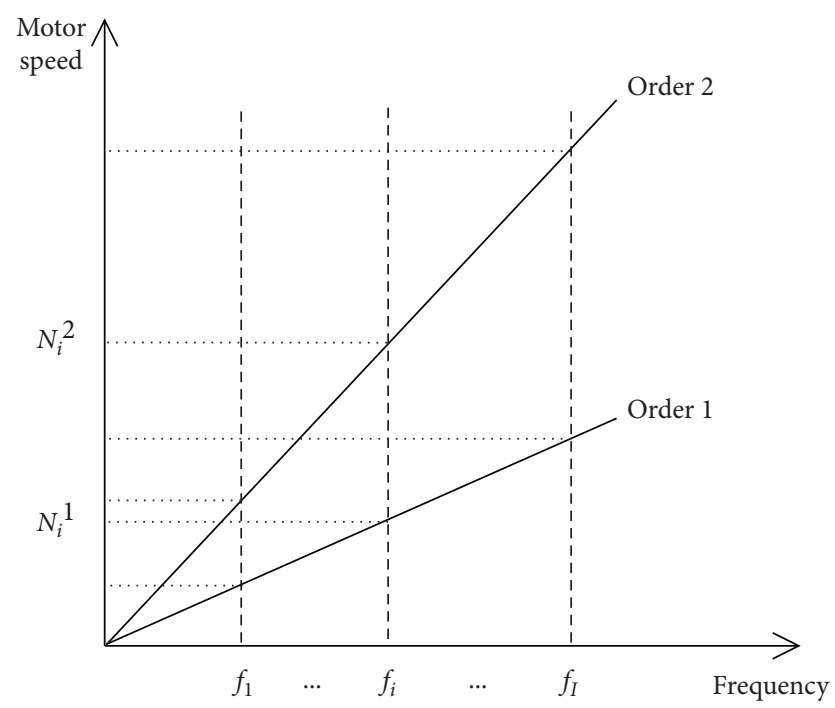

Figure 5: Schematic diagram of the additional steady-speed operation condition determination method.

simulation. Spring elements are used to model the suspension cushions, with the stiffness coefficients of each element set as the static stiffness coefficients of the suspension cushions.

A force treatment program "Force_gene.exe" is worked out to automatically generate order forces, match them with the FE geometry, and output a load file in the format of ".unv" which then can be imported into LMS Virtual Lab for vibration simulation. The program is much more efficient and error-less than loading manually, as the interpolation and loading of the order forces of all 48 stator teeth and 6 bearing holes can be completed by just running the program in seconds. It needs to be noted that the load on each stator tooth obtained by interpolation calculation is still a concentrated force. The code "Force_gene.exe" uniformly decomposed the concentrated force into dozens of point forces distributed on the tooth surface .

5.2. Acoustic Simulation. The boundary element method (BEM) [19] is a native method for simulation acoustic wave problems, especially for exterior acoustic problems. To overcome drawbacks of the conventional BEM, such as efficiency and large memory consumption, fast accelerated BEM has been proposed and applied for large-scale acoustic problems [20, 21]. In this work, acoustic transfer vector (ATV) from the vibration of the powertrain housing to sound pressure response is calculated by the acoustic FE simulation in the software LMS Virtual Lab. ATV can be regarded as the linear input-output transfer relation between the housing vibration and the response point of the sound field, which can be expressed by the following equation:

$$
p(\omega)=\langle\operatorname{ATV}(\omega)\rangle\left\{V_{n}(\omega)\right\}
$$

$\left\{V_{n}(\omega)\right\}$ is the normal vibration velocity matrix of the powertrain housing, $p(\omega)$ denotes the sound pressure of the sound field response point, and $\langle\operatorname{ATV}(\omega)\rangle$ represents the
ATV matrix. The ATV matrix depends on the housing geometry, acoustic impedance at the structure-air interface, acoustic field response position, acoustic signal frequency, and acoustic medium parameters, but it is not related to the surface vibration velocity of the structure. Therefore, ATV can be calculated without the presence of vibration velocity. For the electric powertrain system, NVH simulation analysis is usually required for different operating conditions, such as full-throttle acceleration, half throttle acceleration, and coasting deceleration. For acoustic simulation under multiple operating conditions, the ATV method is more efficient than the direct vibroacoustic FE method. Instead of calculating the sound pressure directly for multiple rounds, the former only needs one round of acoustic FE simulation as the ATV keeps invariable for different operating conditions.

\section{Results and Discussion}

6.1. Bearing Forces. The electromagnetic loads of the motor include two parts, namely, the electromagnetic forces on the teeth and the torque ripple on the rotor. In addition to the tooth order forces discussed in Section 4.1, the torque ripple on the motor rotor also contains the order components in multiples of the number of poles, such as order 8 and order 48. The harmonic torques can be also transmitted to the powertrain housing through the bearings and cause vibration and noise. If one needs to take into account the contribution of the torque ripple on bearing forces, the timedomain signal of the rotor torque obtained by electromagnetic simulation should be adopted in the multi-body dynamic model in Section 4.2. Figure 6 illustrates the amplitude curves of the order forces at one bearing of the input shaft of the reducer, including the orders caused by the electromagnetic torque ripple, i.e., order 8 and order 48 , and those caused by meshing gears. The curve $O_{i j}$ in the figure denotes the $j$-th order force caused by the $i$-th pair of meshing gears. Each curve in the figure denotes the amplitude of the vector sum of the order force, which has been converted into A-weighted level in decibel, with the level $F_{L}$ calculated with equation (2). $F(n)$ is the force amplitude corresponding to rotor speed $n$, and the reference value of the force $F_{0}=1 N$ :

$$
F_{L}=20 \log \left[\frac{F(n)}{F_{0}}\right] .
$$

Figure 6 indicates that the bearing forces caused by the motor torque ripple (orders 8 and order 48) are more than $30 \mathrm{~dB}$ (A) smaller than the gear-meshing order forces, which means that the sound pressure levels (SPL) caused by the former should be much smaller than the latter. It should be noted that the SPLs caused by the electromagnetic forces on the stator teeth could often reach or even exceed those caused by meshing gears, implying that the electromagnetic noise caused by the torque ripple should be a negligible value compared to that caused by the electromagnetic forces on the stator teeth. In the following $\mathrm{NVH}$ simulation, constant torques are used for inputs in multi-body dynamic analysis with torque ripples neglected since the overlooking of the 


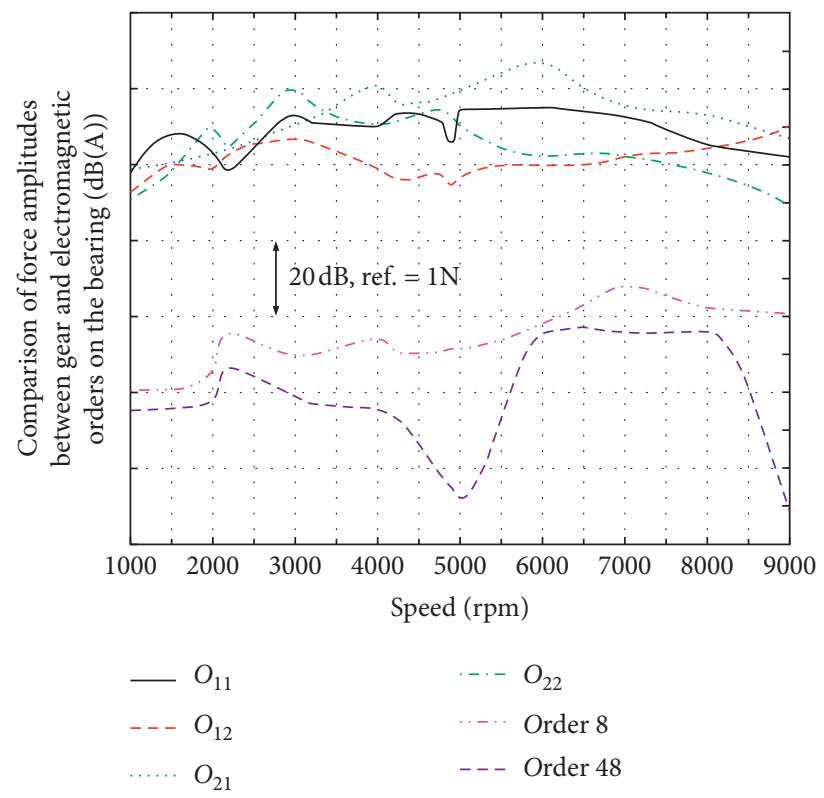

FIGURE 6: Comparison of force amplitudes between gear and electromagnetic orders on the bearing.

motor torque ripple does not result in significant loss in simulation accuracy of electromagnetic noise.

6.2. Experiment Verification and Analysis. The simulated vibration and noise results are compared with the NVH test data for verification. The test is conducted in a semi-anechoic laboratory, and the electric powertrain is installed on the test bench through the suspension system as shown in Figure 7 . Four microphones are located 1 meter away from the powertrain housing in different directions, i.e., the front, back, right, and above, and a 3-axis acceleration sensor is positioned on the powertrain housing. The vibration and sound pressure signals are recorded under the full-throttle acceleration condition.

The vibration acceleration at the housing and the average SPLs of the 4 microphones are presented in Figure 8, with the vibration acceleration denoting the vector sum of the signals of the 3-axis acceleration sensor. As can be seen, the simulation curves and the experiment results show good consistency. The relative speed deviation of any local peak $\left|\delta_{r}\right|$ is below $8 \%$, with $\left|\delta_{r}\right|$ calculated by formula (3), where $N_{\mathrm{CAE}}$ is the motor speed corresponding to the local peak value on the simulation curve and $N_{\text {test }}$ is the motor speed corresponding to the local peak value on the experiment curve. The error of the average SPL is mostly below $10 \mathrm{~dB}(\mathrm{~A})$, with the peak error of the 48 th order around $7200 \mathrm{rpm}$ being about $1 \mathrm{~dB}(\mathrm{~A})$ and the peak error of the order $O_{11}$ around $6800 \mathrm{rpm}$ being about $-8 \mathrm{~dB}(\mathrm{~A})$ :

$$
\delta_{r}=\frac{N_{\mathrm{CAE}}-N_{\text {test }}}{N_{\text {test }}}
$$

The maximum peaks on the 48-order vibration and noise curves appear near $7000 \mathrm{rpm}$, and the response

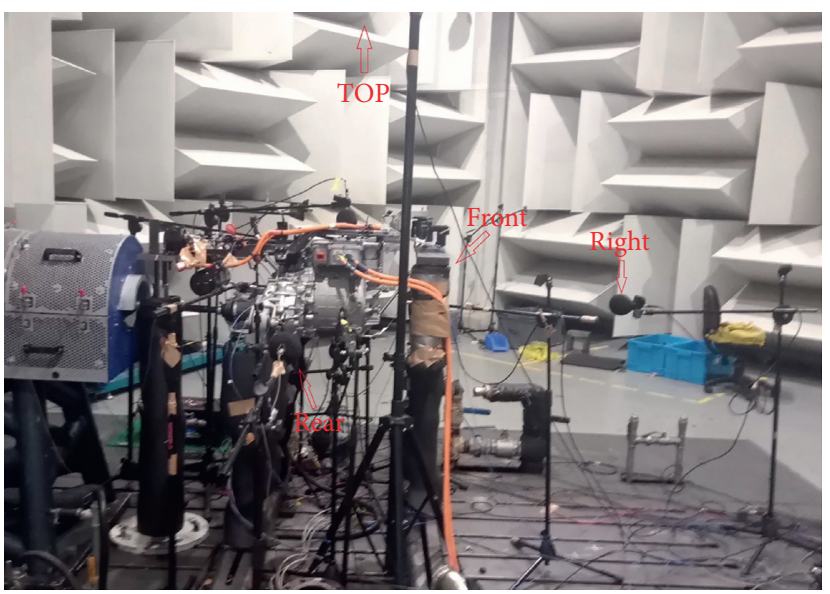

FIGURE 7: NVH test of the powertrain in a semianechoic chamber.

frequency is about $5600 \mathrm{~Hz}$ which is highly consistent with the resonant frequency of the "breathing" mode of the motor stator $(n=0, f 5600 \mathrm{~Hz})$, as presented in Figure 4 . This strong peak appears at around $5600 \mathrm{~Hz}$ due to the following two factors. On the one hand, the spatial zeroorder "force pattern" $(n=0, f 5600 \mathrm{~Hz})$ of the 48 th order electromagnetic force matches well with the stator "breathing" mode in both shape and frequency, leading to strong resonance; on the other hand, for the "breathing" mode, the normal velocity of the stator is in the same phase, which means it has strong acoustic radiation efficiency. The results indicate that accurate simulation of the stator modes is very important for the calculation of motor-related noise, reinforcing the significance of the modal calibration in Section 3. 


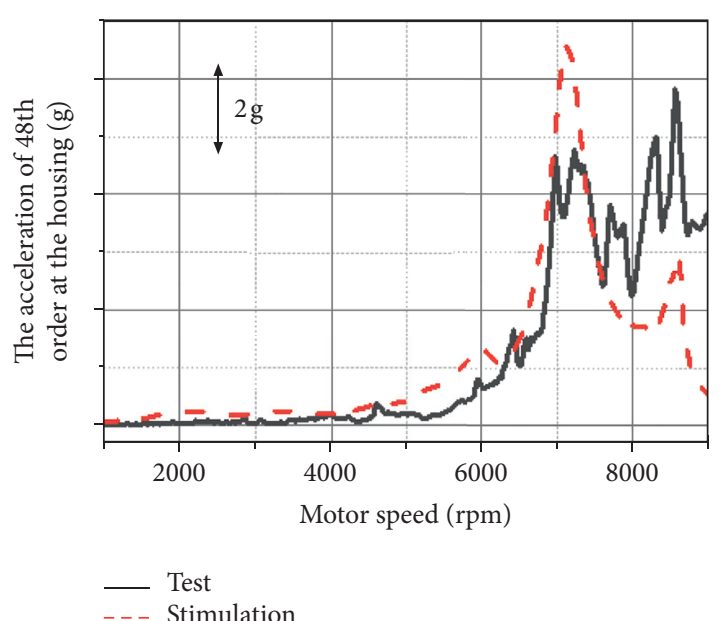

(a)

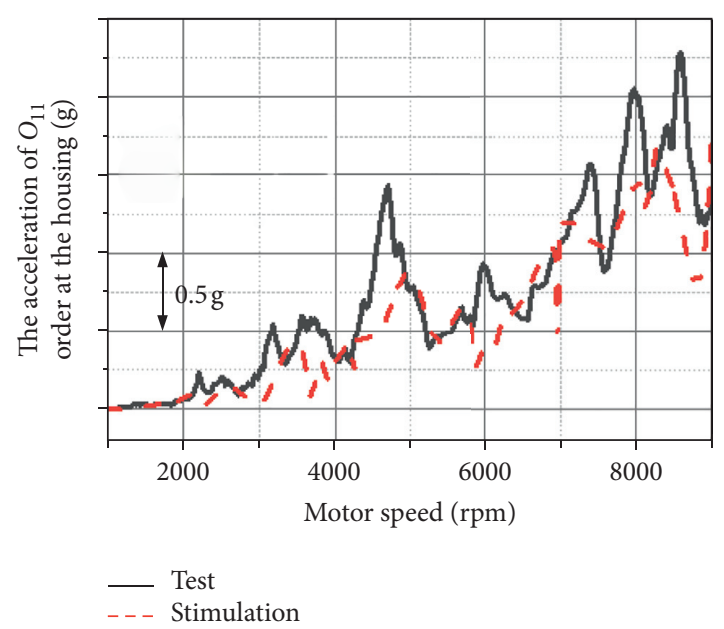

(c)

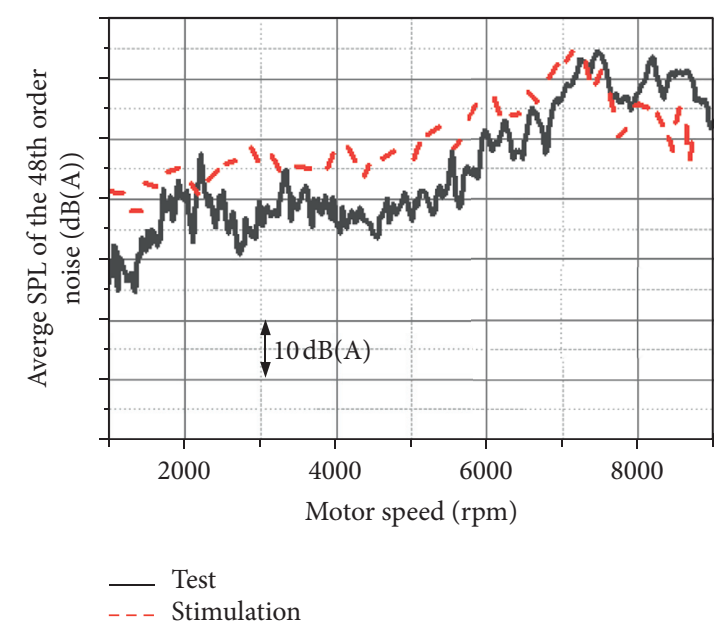

(b)

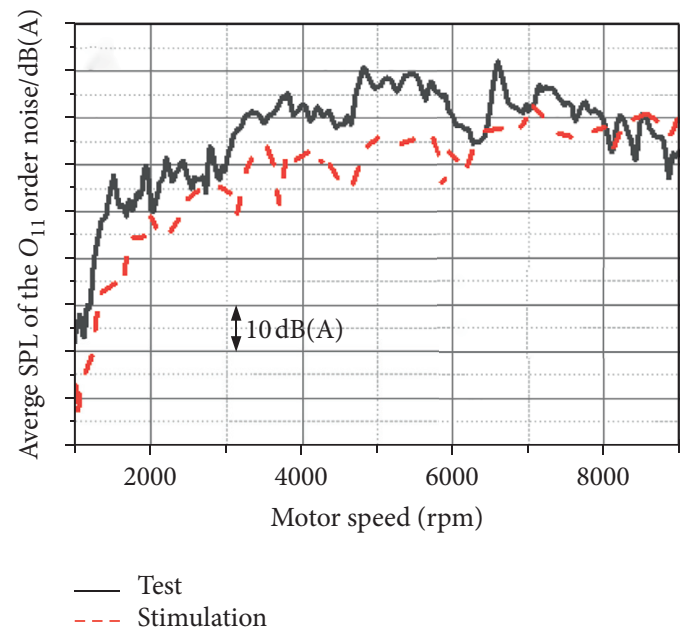

(d)

Figure 8: Comparison between simulation and test: (a) the acceleration of the 48th order at the housing, (b) average SPL of the 48th order noise, (c) the acceleration of the $O_{11}$ order at the housing, and (d) average SPL of the $O_{11}$ order.

\section{Conclusions}

This paper presents a method of NVH simulation analysis for the electric powertrain system under speed-varying conditions. Modal correlation analysis is performed to calibrate the natural modes of the motor stator and improve NVH simulation accuracy.

The calibrated simulated modes are in good agreement with the experimental modes. For the four modes of interest, the frequency errors are within $6.4 \%$, and the MACs are not less than 0.6.

The computed bearing forces show that electromagnetic noise caused by the torque ripple would be a negligible value compared to that caused by the electromagnetic forces on the stator teeth. When multi-body dynamic simulation is used to calculate the bearing forces, ignoring the torque ripple of the motor rotor does not lead to significant loss in electromagnetic SPLs.

The vibration and sound results obtained by simulation and the test are in good agreement. The relative frequency deviation of local peaks between simulation and test curves is less than $8 \%$. The peak error of the motor of 48 -order SPL is about $1 \mathrm{~dB}(\mathrm{~A})$ and that of the order $O_{11}$ is about $-8 \mathrm{~dB}(\mathrm{~A})$.

The 48-order whine noise is strongly related to the breathing mode of the stator. When the circumferential 0order component of the 48th-order electromagnetic force coincides with the stator breathing mode in space and frequency, the vibration and sound curves show strong local resonance peaks.

The influence of model parameters on simulation accuracy should be an interesting research topic in the future.

\section{Data Availability}

The data are not freely available due to the requirement of commercial confidentiality.

\section{Conflicts of Interest}

The authors declare that there are no conflicts of interest regarding the publication of this paper. 


\section{References}

[1] P. Pellerey, V. Lanfranchi, and G. Friedrich, "Coupled numerical simulation between electromagnetic and structural models. Influence of the supply harmonics for synchronous machine vibrations," IEEE Transactions on Magnetics, vol. 48, no. 2, pp. 983-986, 2012.

[2] Q. Kang, P. Gu, C. Gong, and S. Zuo, Test and Analysis of Electromagnetic Noise of an Electric Motor in a Pure Electric Car, SAE Technical Paper, Grand Rapids, MI, USA, 2019.

[3] L. Humbert, P. Pellerey, and S. Cristaudo, "Electromagnetic and structural coupled simulation to investigate $\mathrm{NVH}$ behavior of an electrical automotive powertrain," SAE International Journal of Alternative Powertrains, vol. 1, no. 2, pp. 395-404, 2012.

[4] Y. Fang and T. Zhang, "Vibroacoustic characterization of a permanent magnet synchronous motor powertrain for electric vehicles," IEEE Transactions on Energy Conversion, vol. 33, no. 1, pp. 272-280, 2017.

[5] O. Harris, P. Langlois, and A. Gale, Electric Vehicle Whine Noise-Gear Blank Tuning as an Optimization Option, pp. 64-73, Gear Technology, Chicago, IL, USA, 2019.

[6] P. Yu, S.-Y. Chen, T. Zhang, and R. Guo, "Vibration response of an EV power train under mechanical-electromagnetic excitation," Vibration and Shock, vol. 35, no. 13, pp. 99-105, 2016.

[7] P. Yu, F.-F. Chen, T. Zhang, and R. Guo, "Vibration characteristics analysis of a central-driven electric vehicle powertrain," Vibration and Shock, vol. 34, no. 1, pp. 44-48, 2015.

[8] P. Vijayraghavan and R. Krishnan, "Noise in electric machines: a review," IEEE Transactions on Industry Applications, vol. 35, no. 5, pp. 1007-1013, 1999.

[9] J. F. Gieras, C. Wang, and J. C. Lai, Noise of Polyphase Electric Motors, CRC Press, Boca Raton, FL, USA, 2018.

[10] N. Chandrasekhar, C. Tang, N. Limsuvan et al., Current Harmonics, Torque Ripple and Whine Noise of Electric Machine in Electrified Vehicle Applications, SAE Technical Paper, Detroit, MI, USA, 2017.

[11] T. C. Lim and R. Singh, A Review of Gear Housing Dynamics and Acoustics Literature, NTRS Report NAS 1.26:183110, Ohio State University, Columbus, OH, USA, 1988.

[12] W. Deng, S. Zuo, H. Sun, S. Wu, and G. Zhang, "Modal analysis of a claw-pole alternator considering orthotropy of the stator core and windings," Vibration and Shock, vol. 36, no. 12, pp. 43-49, 2017.

[13] S. Zuo, Y. Zhang, J. Yan, G. Zhang, F. Lin, and S. Wu, "Optimization of vibration and noise in permanent magnet synchronous motor considering stator anisotropy," Journal of Xi'an Jiaotong University, vol. 51, no. 5, pp. 60-68, 2017.

[14] P. Millithaler, É. Sadoulet-Reboul, M. Ouisse, J.-B. Dupont, and N. Bouhaddi, "Structural dynamics of electric machine stators: modelling guidelines and identification of three-dimensional equivalent material properties for multi-layered orthotropic laminates," Journal of Sound and Vibration, vol. 348, pp. 185-205, 2015.

[15] Q. Deng, "A high efficiency NVH simulation methodology for vehicle driving motor based on electromagnetic force approximation," Journal of Applied Acoustics, vol. 38, no. 6, pp. 932-938, 2019.

[16] Y. Chen, Z. Zhu, and S. Ying, Analysis and Control of Electric Motor Noise, Zhejiang University Press, Hangzhou, China, 1987.

[17] G. Zheng, X. Huang, and D. Guo, "Effects of flexible gearbox body on dynamic meshing performance of its gear pair,"
Journal of Vibration and Shock, vol. 36, no. 13, pp. 140-145, 2017.

[18] P. K. Singh, Study of Effect of Variation in Micro-Geometry of Gear Pair on Noise Level at Transmission, SAE Technical Paper, Pune, India, 2015.

[19] L. G. Copley, "Fundamental results concerning integral representations in acoustic radiation," The Journal of the Acoustical Society of America, vol. 44, no. 1, pp. 28-32, 1968.

[20] H. Wu, Y. Liu, and W. Jiang, "A fast multipole boundary element method for 3D multi-domain acoustic scattering problems based on the Burton-Miller formulation," Engineering Analysis with Boundary Elements, vol. 36, no. 5, pp. 779-788, 2012.

[21] H. Wu, Y. Liu, and W. Jiang, "A low-frequency fast multipole boundary element method based on analytical integration of the hypersingular integral for 3D acoustic problems," Engineering Analysis with Boundary Elements, vol. 37, no. 2, pp. 309-318, 2013. 